\title{
Morphological changes in convection-diffusion-limited deposition
}

\author{
Takashi Nagatani \\ College of Engineering, Shizuoka University, Hamamatsu 432, Japan \\ Francesc Sagués \\ Department de Quimica-Fisica, Universitat de Barcelona, Diagonal 647, E-08028 Barcelona, Spain
}

(Received 10 September 1990)

\begin{abstract}
The effect of hydrodynamic flow upon diffusion-limited deposition on a line is investigated using a Monte Carlo model. The growth process is governed by the convection and diffusion field. The convective diffusion field is simulated by the biased-random walker resulting from a superimposed drift that represents the convective flow. The development of distinct morphologies is found with varying direction and strength of drift. By introducing a horizontal drift parallel to the deposition plate, the diffusion-limited deposit changes into a single needle inclined to the plate. The width of the needle decreases with increasing strength of drift. The angle between the needle and the plate is about $45^{\circ}$ at high flow rate. In the presence of an inclined drift to the plate, the convectiondiffusion-limited deposit leads to the formation of a characteristic columnar morphology. In the limiting case where the convection dominates, the deposition process is equivalent to ballistic deposition onto an inclined surface.
\end{abstract}

\section{INTRODUCTION}

Recently there has been increasing interest in a variety of nonequilibrium aggregation and deposition models such as the diffusion-limited aggregation (DLA) model and ballistic deposition. ${ }^{1-11}$ A variety of computer simulations have been carried out to investigate the relationships between the cluster geometry and growth mechanisms. The structure of the aggregates strongly depends on the dynamics of the growth process. The most simple growth models are ballistic deposition and diffusionlimited aggregation. ${ }^{11}$ The ballistic deposition model provides a basis for understanding deposition processes used to prepare a wide variety of thin-film devices. The diffusion-limited-aggregation model presents a prototype of the pattern formation of diffusive systems including the electrochemical deposition, crystal growth, viscous fingering, and dielectric breakdown. ${ }^{11}$ Several simple generalizations of the DLA model have been carried out to take into account particle drift, ${ }^{12-14}$ sticking probability, ${ }^{15,16}$ surface tension, ${ }^{17-19}$ finite viscosity ratio, ${ }^{20-23}$ multiparticle effect, ${ }^{24,25}$ and lifetime effects. ${ }^{26}$ Meakin $^{12}$ investigated the effect of particle drift on DLA with the use of a biased-random walker and found a crossover phenomenon from the DLA fractal to the dense aggregation. Furthermore, the structural phase transition between the needle structure and the dense aggregate was found by inverting the direction of the radial drift. ${ }^{14}$ The morphological changes from the dense deposit to the columnar structure was shown by simulations of ballistic deposition onto an inclined surface. ${ }^{27}$ Xiao, Alexander, and Rosenberger ${ }^{28}$ simulated the morphological evolution of crystals growing under the influence of a uniform drift. It has been found that the drift has an important effect on the morphology of the deposit. Also, in the electrochemical deposition experiments, it has been suggested that the convection may have an important effect on the morphology. ${ }^{29,30}$ However, there is an open question whether or not the direction of drift has an effective effect on the morphology of the deposit.

In this paper we study the morphological evolution of the convection-diffusion-limited aggregation deposited on a line by changing direction of the hydrodynamic flow. The growth process is governed by the convection and diffusion fields. We simulate the convective diffusion field by using a biased-random walker resulting from a superimposed drift which represents the convective flow. The distinct morphologies are found with varying direction and strength of flow. Especially the introduction of a horizontal flow parallel to the deposition plate changes the morphologies of diffusion-limited deposits into a single needle inclined to the plate. We find that the width of the needle decreases with increasing strength of flow and the angle between the needle and the plate is about $45^{\circ}$ at high flow rate. Furthermore, it is found that the introduction of an inclined flow to the plane also leads in this case to the formation of a columnar morphology. In the limiting case in which the convection dominates, it is shown that the deposition process approaches the ballistic deposition onto an inclined surface. ${ }^{27,31,32}$ We also calculate the growth probability distributions on the needles and study the effect of the flow on the growth probability distribution.

The organization of the paper is as follows. In Sec. II we present the basic equation of the convective-diffusion field. We give the discrete version of the convectivediffusion equation for simulation. In Sec. III we present the computational results. The development of the distinct morphologies is shown in the figures. In Sec. IV we show the growth probability distributions of the needles 
under various flow conditions. The relationship between the tip velocity and the inclined angle of the needle is discussed. Section V presents the summary.

\section{BASIC EQUATION AND MODEL}

We consider the deposition process growing on a surface in two dimensions. The pattern formation is governed by the convective-diffusion field of a component outside the growing deposit without surface kinetics. Figure 1 shows a schematic representation of the problem. The concentration $C$ of the diffusing component with convection satisfies the convective diffusion equation under the quasistationary approximation

$$
u_{x} \frac{\partial C}{\partial x}+u_{y} \frac{\partial C}{\partial y}=D \frac{\partial^{2} C}{\partial x^{2}}+D \frac{\partial^{2} C}{\partial y^{2}},
$$

where $u_{x}$ and $u_{y}$ are the $x$ and $y$ components of the velocity, $D$ is the diffusion constant. The velocity is assumed to be constant. We consider the convective-diffusion field within a square of dimension $L \times L$. The deposit grows from the lower boundary towards the upper boundary. The boundary conditions in the horizontal direction are periodic in order to simulate an infinite half-plane. The concentration on the surface of the deposit is zero, and the concentration at the upper boundary is constant $C_{0}$. To cast the convective-diffusion equation in dimensionless form, we use the following units: $C_{0}$ for the concentration, $a$ for distance. Then Eq. (1) becomes

$$
\hat{U}_{x} \frac{\partial \hat{C}}{\partial \hat{x}}+\hat{U}_{y} \frac{\partial \hat{C}}{\partial \hat{y}}=\frac{\partial^{2} \hat{C}}{\partial \hat{x}^{2}}+\frac{\partial^{2} \hat{C}}{\partial \hat{y}^{2}},
$$

where $\hat{U}_{x(\text { or } y)}=u_{x(\text { or } y)} a / D, \hat{C}=C / C_{0}, \hat{x}=x / a$, and $\hat{y}=y / a$. Careted variables are all dimensionless. The dimensionless velocity $\widehat{U}$ represents the Peclet number. The boundary conditions are given by $\hat{C}=0$ on the surface of the deposit, and $\hat{C}=1$ on the upper boundary. Now we consider the simulation of the aggregation pro-

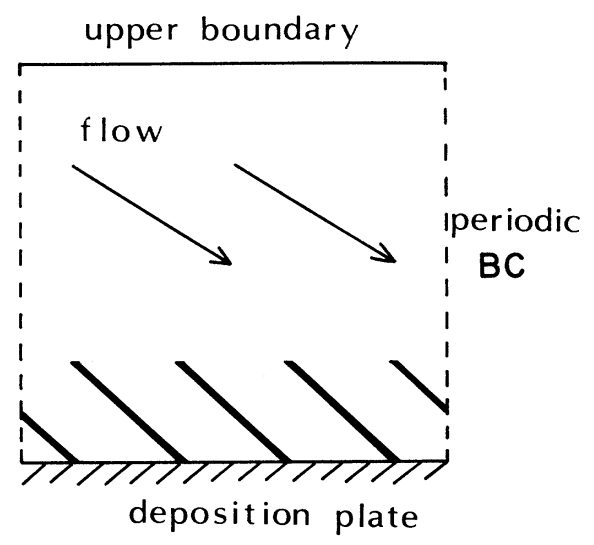

FIG. 1. Schematic representation of the diffusion-limited aggregation in the presence of the hydrodynamic flow. Particles are introduced one at a time at a randomly chosen point on the upper boundary. The particle performs a biased-random walk following the hydrodynamic flow. The lateral boundary is periodic. (BC represents boundary condition.) cess following Eq. (2). The convective diffusing particles undergo random walks except for a bias resulting from the convection. The probability $p(i, j)$ that the biased random walker visit the site $(i, j)$ satisfies the equation

$$
\begin{aligned}
p(i, j)= & {\left[\frac{1-P_{x}-P_{y}}{4}\right] p(i+1, j) } \\
& +\left[P_{x}+\frac{1-P_{x}-P_{y}}{4}\right] p(i-1, j) \\
& +\left[\frac{1-P_{x}-P_{y}}{4}\right] p(i, j+1) \\
& +\left[P_{y}+\frac{1-P_{x}-P_{y}}{4}\right] p(i, j-1),
\end{aligned}
$$

where

$$
P_{x}=\frac{\hat{U}_{x}}{1+\hat{U}_{x}+\hat{U}_{y}}, \quad P_{y}=\frac{\hat{U}_{y}}{1+\hat{U}_{x}+\hat{U}_{y}} .
$$

We choose the reference length $a$ to be the lattice spacing. $P_{x}$ and $P_{y}$ represent the horizontal and the vertical drifts resulting from the convective flow. The horizontal and vertical drifts are obtained from the velocity of the convection via Eq. (3b). The drift is defined by Eq. (3b). The convective diffusion field can be simulated by using a biased-random walker resulting from a superimposed drift which represents the convective flow.

One can also use the other method proposed by Xiao, Alexander, and Rosenberger. ${ }^{28}$ In their simulation method, the drift is superimposed on each step of random walk. The results of their simulation are almost the same as our results. However, in their case, the drift $\hat{U}_{x \text { (or } y \text { ) }}$ must always be used as an integer. Instead, the simulation with use of Eq. (3) is possible for any drifts. The simulation by Eq. (3) is thus more general than that by Xiao, Alexander, and Rosenberger. ${ }^{28}$ We use Eq. (3) for our simulation here.

\section{SIMULATION RESULT}

We summarize the algorithm of the simulation process (see Fig. 1). We consider the diffusive system with both horizontal and vertical drift. The simulation of the DLA cluster formation in the presence of the horizontal and vertical drift of particles is carried out with the use of a simple square lattice. We consider a subset of the square lattice enclosed by square with $300 \times 300$ (units). We start out with an occupied plate (shown by the shaded line in Fig. 1) on the bottom of the square. The lateral boundary is periodic. Particles are introduced one at a time at a randomly chosen point on the upper boundary. We allow particles to follow horizontal and vertical biased random trajectories in the vicinity of the original occupied plate. For a horizontal drift, particles perform a horizontally biased random walk with drift probability $P_{x}$ toward the horizontal direction. For both horizontal and vertical drift, particles perform a biased Brownian motion with drift probability $\boldsymbol{P}_{\boldsymbol{y}}$ along the vertical direc- 
tion (taken arbitrarily positive when directed towards the plate) and with drift probability $P_{x}$ along the horizontal direction (positive to the right). The particle continues to move until it either reaches a point adjacent to a site already occupied by a particle or until it reaches the upper boundary. When the particle reaches a point adjacent to a site already occupied by a particle, it sticks on the aggregate. When the particle reaches the upper boundary, the random walker is annihilated. We repeat the procedure. The clusters are grown until the height reaches 150 units. We have carried out calculations for a range of $P_{x}$ values $\left(0<P_{x}<1\right)$ under the pure horizontal drift. We have also performed calculations for a range of $P_{x}$ and $P_{y}$ values $\left(0<P_{x}+P_{y}<1\right)$ in the presence of the horizontal and the vertical drift. Figure 2 shows the results obtained for pure horizontal drifts by using the procedures outlined above. Figures 2(a)-2(d) show, respectively, the clusters for $P_{x}=0.05,0.1,0.2$, and 0.5. By introducing the horizontal drift, the overall shape of the deposit changes into a single tree. As the horizontal drift
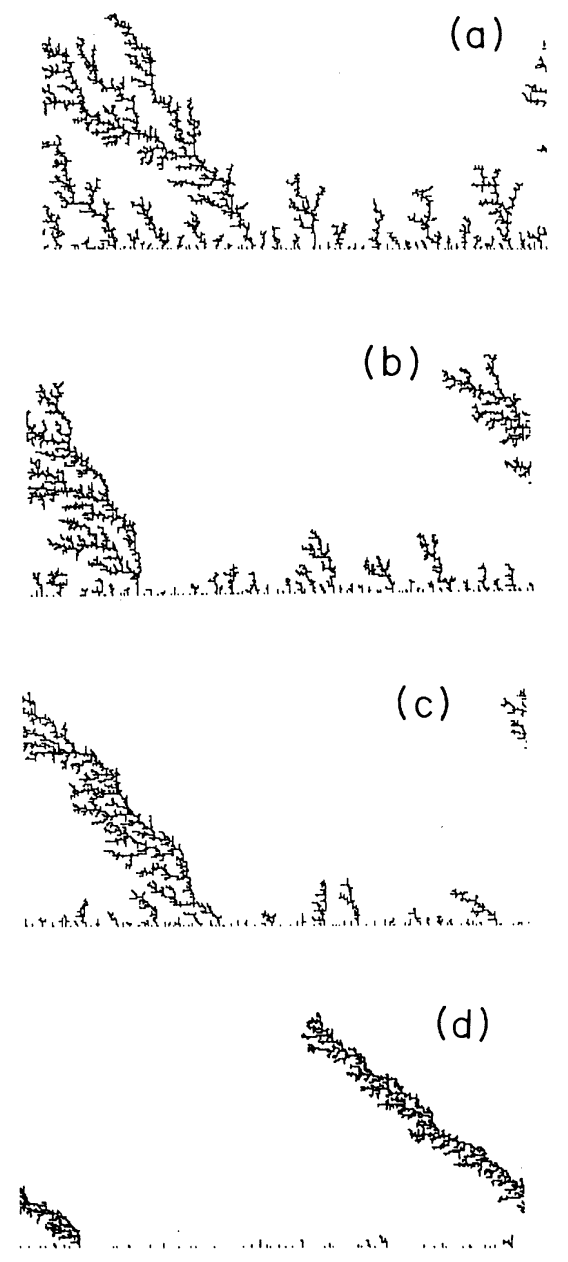

FIG. 2. Typical patterns grown under pure horizontal drifts. By introducing the horizontal drift, the DLA changes into a single needle structure. (a) $P_{x}=0.05$. (b) $P_{x}=0.1$. (c) $P_{x}=0.2$. (d) $P_{x}=0.5$. increases, the shape of the cluster becomes a less ramified structure and changes finally into a single needle inclined against the drift. In the limit of $P_{x} \rightarrow 0$, the cluster approaches to the DLA fractal. The introduction of the horizontal drift has a strong effect upon the structure of the cluster. The horizontal drift induces a strong screening. The screening effect prevents particles to go in the inner part of the deposit. The tip of the cluster grows mainly. The single needle forming under the horizontal drift should be compared with the dense structure forming in the presence of an attractive vertical drift. ${ }^{33}$ As a second step, we study the combined effect of the horizontal drift and the vertical drift upon the DLA deposition. First, we vary the horizontal drift under the condition of constant vertical drift. Figures 3(a)-3(d) show, respectively, the patterns obtained from $P_{x}=0.1: P_{y}=0.1$, $P_{x}=0.3: P_{y}=0.1, P_{x}=0.5: P_{y}=0.1$, and $P_{x}=0.7: P_{y}=0.1$.
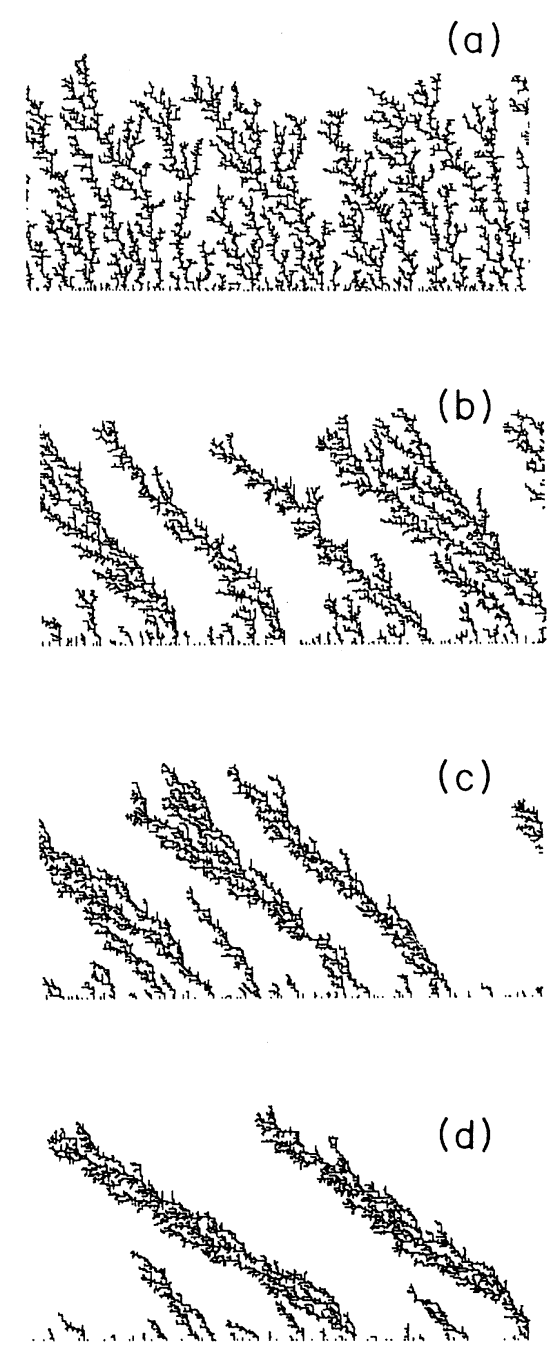

FIG. 3. Typical patterns grown by varying the horizontal drift under the condition of constant vertical drift. With increasing horizontal drift, the pattern of the deposits changes into the columnar morphology. (a) $P_{x}=0.1: P_{y}=0.1$. (b) $P_{x}=0.3: P_{y}=0.1$. (c) $P_{x}=0.5: P_{y}=0.1$. (d) $P_{x}=0.7: P_{y}=0.1$. 
With increasing horizontal drift, the pattern of the deposits changes into the columnar morphology. The width and the number of the columns decrease with increasing horizontal drift. In the limit of no horizontal drift, the pattern of the deposits is similar to the dense structure in the DLA with pure vertical drift. ${ }^{33}$ On the other hand, approaching the limit of $P_{x}+P_{y}=1$, the pattern adopts the columnar morphology in the ballistic model onto an inclined surface. ${ }^{27,31}$ For further use we call the ballistic model onto an inclined surface the inclined ballistic model. Second, we vary the vertical drift under the condition of the constant horizontal drift. Figures 4(a)-4(d) show, respectively, the patterns obtained under the following conditions: $P_{x}=0.5: P_{y}=0.5, \quad P_{x}=0.5: P_{y}=0.3$, $P_{x}=0.5: P_{y}=0.2$, and $P_{x}=0.5: P_{y}=0.1$. With decreasing vertical drift, the pattern of the deposits changes again into the columnar structure. The number of the columns

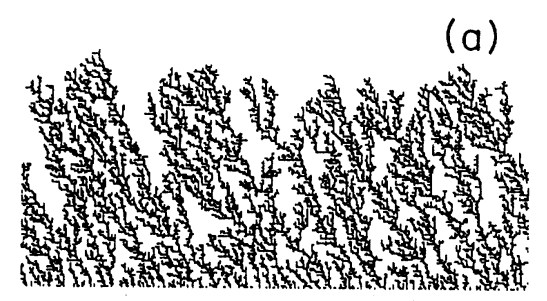

(b)
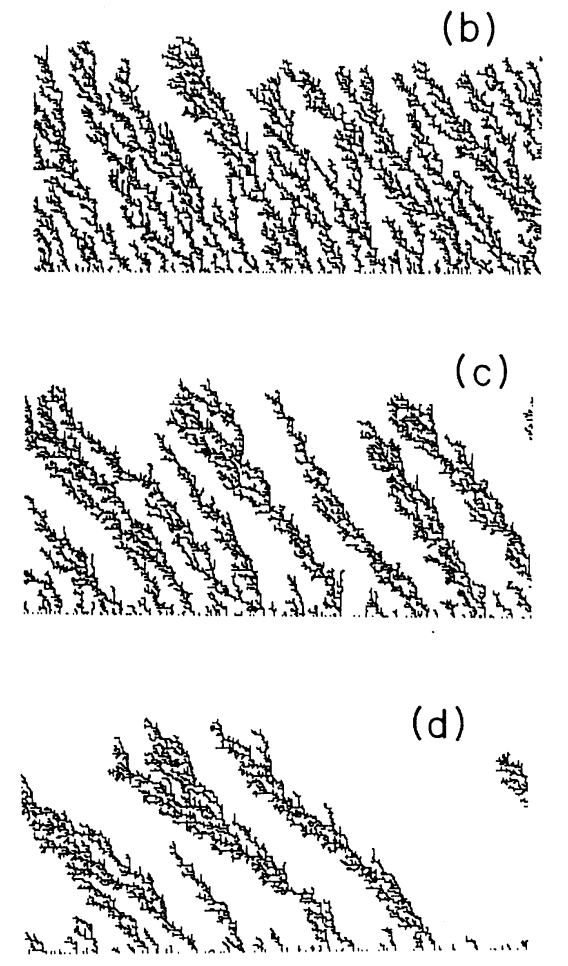

FIG. 4. Typical patterns grown by varying the vertical drift under the condition of constant horizontal drift. With decreasing vertical drift, the pattern of the deposits changes into the columnar morphology. (a) $P_{x}=0.5: P_{y}=0.5$. $P_{x}=0.5: P_{y}=0.3$. (c) $P_{x}=0.5: P_{y}=0.2$. (d) $P_{x}=0.5: P_{y}=0.1$.
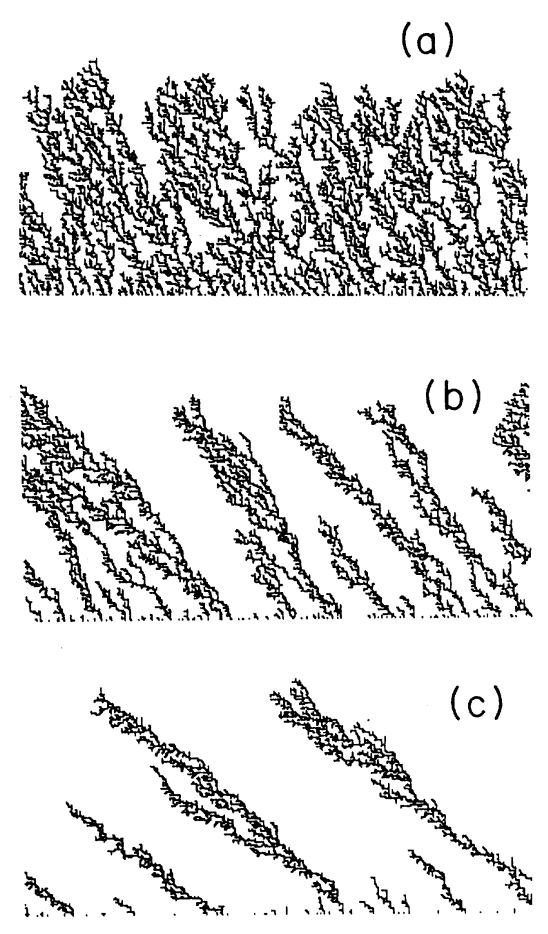

FIG. 5. Typical patterns grown by the inclined ballistic model. (a) $P_{x}=0.5: P_{y}=0.5$. $P_{x}=0.9: P_{y}=0.1$.

(b) $P_{x}=0.75: P_{y}=0.25$. (c)

decreases with the vertical drift. For comparison, we show the patterns obtained from the pure ballistic model in Fig. 5. Figures 5(a)-5(c) show, respectively, the patterns for $P_{x}=0.5: P_{y}=0.5, \quad P_{x}=0.75: P_{y}=0.25$, and $P_{x}=0.9: P_{y}=0.1$. In the limit where the convection dominates the diffusion, the inclined ballistic model is obtained. In summary, by adding the vertical drift to the horizontal drift, the structure of the pattern with the single needle changes into the columnar morphology. The

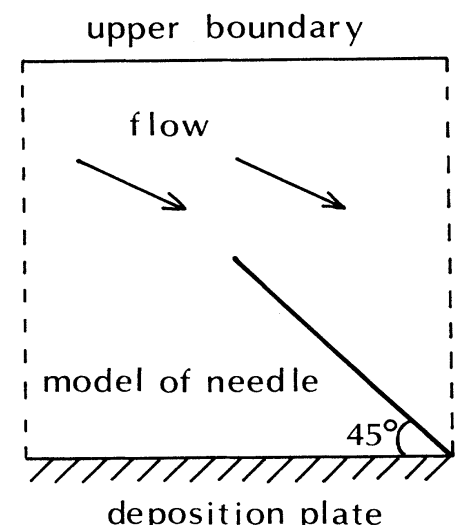

FIG. 6. Model of the needle for calculating the growth probability distribution under various drift conditions. The needle is modeled by the line with the inclined angle $45^{\circ}$. The growth probability distributions on the line are calculated with varying strength and direction of the drift. 
direction of the drift has an important effect upon the deposition process.

\section{GROWTH PROBABILITY DISTRIBUTION}

We study the growth probability distributions on the inclined needle under various drift conditions. We dis-
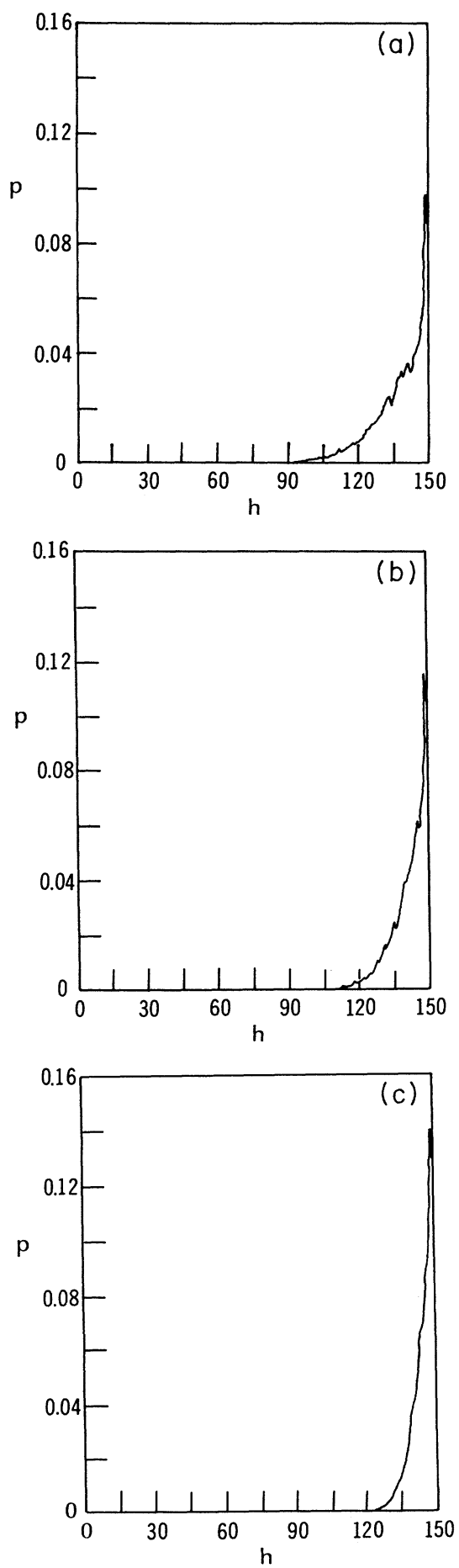

FIG. 7. Growth probability distributions under pure horizontal drifts. (a) $P_{x}=0.3$. (b) $P_{x}=0.5$. (c) $P_{x}=0.7$. cuss the morphology changes between the single needle and the columnar structure. Furthermore, we discuss the relationship between the tip velocity and the inclined angle of the needle. For simplicity, we consider a line with
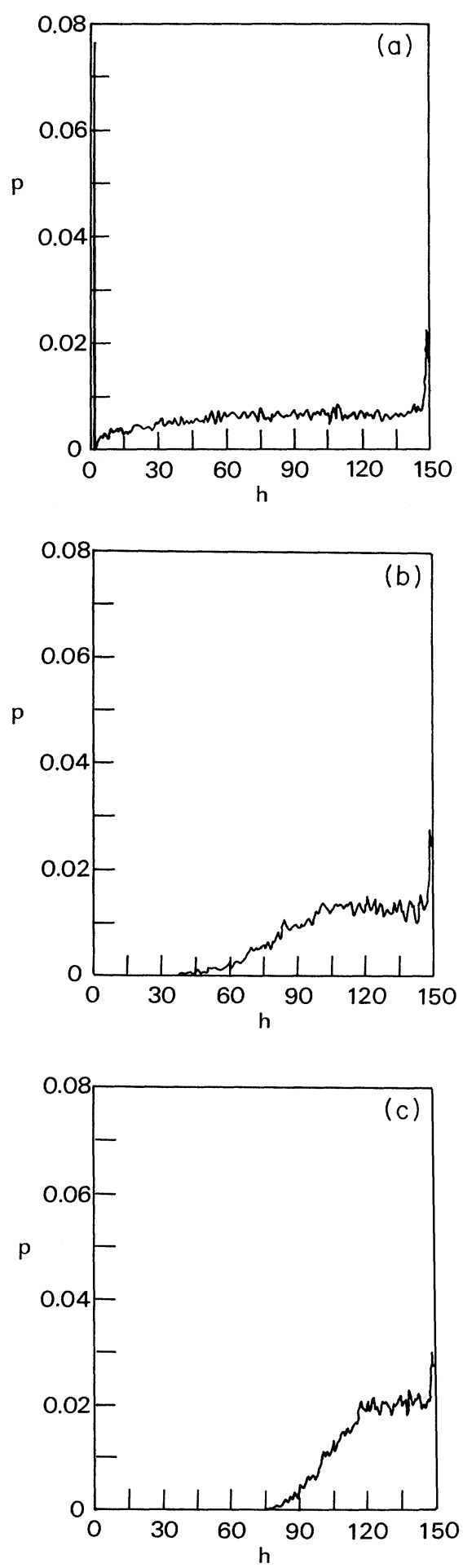

FIG. 8. Growth probability distributions obtained by adding the vertical drift $P_{y}=0.1$ to the horizontal drifts. The growth probability distributions in Figs. $7(a)-7(c)$ change, respectively, into $8(\mathrm{a})-8(\mathrm{c})$. 
TABLE I. Growth probabilities at the tips of the needles for Figs. 7(a)-7(c) and 8(a)-8(c). The comparison of the inclined angle obtained from Eq. (6) with that from the simulation.

\begin{tabular}{lcccc}
\hline \hline & $p_{x}$ & $p_{y}$ & $\tan ^{-1}\left(p_{y} / p_{x}\right)$ & $\phi$ (simulation) \\
\hline Fig. 7(a) & $0.098 \pm 0.01$ & $0.072 \pm 0.01$ & $36.3^{\circ} \pm 7^{\circ}$ & $43^{\circ}$ \\
Fig. 7(b) & $0.115 \pm 0.01$ & $0.094 \pm 0.01$ & $39.3^{\circ} \pm 7^{\circ}$ & $41^{\circ}$ \\
Fig. 7(c) & $0.139 \pm 0.01$ & $0.137 \pm 0.01$ & $44.6^{\circ} \pm 7^{\circ}$ & $47^{\circ}$ \\
Fig. 8(a) & $0.023 \pm 0.01$ & $0.014 \pm 0.01$ & $31.3^{\circ} \pm 7^{\circ}$ & $45^{\circ}$ \\
Fig. 8(b) & $0.027 \pm 0.01$ & $0.023 \pm 0.01$ & $40.4^{\circ} \pm 7^{\circ}$ & $46^{\circ}$ \\
Fig. 8(c) & $0.030 \pm 0.01$ & $0.027 \pm 0.01$ & $42.0^{\circ} \pm 7^{\circ}$ & $43^{\circ}$ \\
\hline \hline
\end{tabular}

an inclined angle $45^{\circ}$ for modeling the inclined needle. Figure 6 shows the model of the needle. The model line is the height 150 with the inclined angle $45^{\circ}$ within the square $300 \times 300$. We calculate the growth probability distributions on the line with varying strength and direction of the drift. Furthermore, we calculate the tip velocity. The growth probability distribution on the model line is calculated as follows. First, particles are introduced one at a time at a randomly chosen point on the upper boundary. The particle performs a biased-random walk following Eq. (3). The particle continues to move until it reaches a point adjacent to the model line, until it lands on the plate, or until it reaches the upper boundary. The sites on the model line have the counter associated to its vertical ordinate. The sites on the plate correspond to $h=1$. The number of times that the particle hits the site is counted. As soon as the particle hits the model line, the particle is removed. When the particle reaches the upper boundary, it is also annihilated. A new particle is introduced at randomly chosen point on the upper boundary. The procedure is repeated up to 10000 times. Thus one can obtain the growth probability distribution on the model line. Figure 7 shows the growth probability distributions for some horizontal drifts. The growth probability distributions in Figs. $7(\mathrm{a})-7(\mathrm{c})$ give, respectively, those for horizontal drift $P_{x}=0.3,0.5$, and 0.7 . With the increasing strength of the horizontal drift, the growth probability distribution rapidly accumulates near the tip of the needle. Only the tip of the needle grows. The inner part of the needle is screened strongly. The structure of the single needle forming under the pure horizontal drift is thus due to the strong screening effect. Furthermore, we study how the growth probability distribution varies by adding the vertical drift to the horizontal drift. Figures 8(a)-8(c) show, respectively, the growth probability distributions for both vertical and horizontal drifts $P_{x}=0.3: P_{y}=01, \quad P_{x}=0.5: P_{y}=0.1, \quad$ and $P_{x}=0.7: P_{y}=0.1$. By adding the vertical drift $P_{y}=0.1$ to the horizontal drifts, the growth probability distributions in Figs. $7(a)-(7 c)$ change into those in Figs. 8(a)-8(c). The screening effects of the horizontal drift are strongly weakened by adding the vertical drift. In the condition of Fig. 8(a), particles can reach the plate. A peak in the growth probability distribution appearing for $h=1$ shows the probability that particles can reach the plate. Actually, a steep change is observed regarding the probability of landing on the plate when increasing the horizontal drift [compare Figs. 8(b) and 8(c) with 8(a)]. This somewhat unrealistic feature of the simulations is probably related to the practical utilization of the model line as it is used here. The introduction of the vertical drift breaks the screening effect. Thus, by adding the vertical drift to the horizontal drift, the pattern changes from the single needle into the columnar morphology. We can explain the morphological changes in terms of the growth probability distribution.

We consider the tip velocity and the inclined angle of the needle. The tip velocity is proportional to the growth probability of the tip. The horizontal velocity $u_{x}$ and the vertical velocity $u_{y}$ at the tip are respectively proportional to the horizontal growth probability $p_{x}$ and the vertical growth probability $p_{y}$ at the tip:

$$
\begin{aligned}
& u_{x} \approx p_{x}, \\
& u_{y} \approx p_{y} .
\end{aligned}
$$

The inclined angle of the needle is given by

$$
\phi=\tan ^{-1}\left(u_{y} / u_{x}\right)=\tan ^{-1}\left(p_{y} / p_{x}\right) .
$$

We show the growth probabilities of the tips for Figs. 7(a)-7(c) and 8(a)-8(c) in Table I. We also compare the inclined angle calculated from Eq. (7) with that from the simulation. The value of the inclined angle derived from Eq. (7) has a slightly smaller value than that from the simulation. The difference may be due to approximating the needle by the inclined line. The fluctuation of the inclined angle may be due to small size of system as it is used here. Meakin ${ }^{27}$ obtained $\phi=33^{\circ}$ for the inclined ballistic model with the inclined angle $90^{\circ}$. This value corresponds to the limit of pure horizontal drift. Our simulation result is not as small as that by Meakin. ${ }^{27}$ This inconsistency is due to small system sizes as it is used here.

\section{SUMMARY}

We investigate the effect of hydrodynamic flow upon the diffusion-limited deposition using a Monte Carlo model. We simulate the pattern formation governed by the convection and diffusion by making use of the biased-random walker. We find that distinct morphologies develop with varying direction and strength of flow. We show that the diffusion-limited deposit changes into a single needle inclined to the plate by introducing a horizontal flow parallel to the deposition plate. The width of the needle decreases with increasing strength of flow. We 
also find that deposits with the typical columnar morphology form in the presence of both horizontal and vertical flow. We calculate the growth probability distribution on the inclined needle. We show that the morphological changes can be explained in terms of the growth probability distribution.

\section{ACKNOWLEDGMENTS}

One of us (F.S.) acknowledges support received from Kyushu Institute of Technology (Japan) and from Direccion General de Investigacion Científica y Técnica (Spain) during the completion of this work.
${ }^{1}$ T. A. Witten and L. M. Sander, Phys. Rev. Lett. 47, 1400 (1981); Phys. Rev. B 27, 5686 (1983).

${ }^{2}$ P. Meakin, Phys. Rev. A 26, 1495 (1983); 27, 604 (1983); 27, 2616 (1983).

${ }^{3}$ Kinetics of Aggregation and Gelation, edited by F. Family and D. P. Landau (North-Holland, Amsterdam, 1984).

${ }^{4}$ On Growth and Form, edited by H. E. Stanley and N. Ostrowsky (Nijhoff, The Hague, 1985).

${ }^{5}$ Fractals in Physics, edited by L. Pietronero and E. Tosatti (North-Holland, Amsterdam, 1986).

${ }^{6}$ H. J. Herrmann, Phys. Rep. 136, 153 (1986).

${ }^{7} \mathbf{P}$. Meakin, in Phase Transitions and Critical Phenomena, edited by C. Domb and J. L. Lebowitz (Academic, New York, 1988), Vol. 12, p. 336.

${ }^{8}$ R. Julien and R. Botet, Aggregation and Fractal Aggregates (World Scientific, Singapore, 1987).

${ }^{9}$ J. Feder, Fractals (Plenum, New York, 1988).

${ }^{10}$ Random Fluctuations and Pattern Growth, edited by H. E. Stanley and N. Ostrowsky (Kluwer Academic, Dordrecht, 1988).

${ }^{11}$ T. Vicsek, Fractal Growth Phenomena (World Scientific, Singapore, 1989).

${ }^{12}$ P. Meakin, Phys. Rev. B 28, 5221 (1983).

${ }^{13}$ R. Kapral, S. G. Whittington, and R. C. Desai, J. Phys. A 19, 1727 (1986).

${ }^{14}$ T. Nagatani, Phys. Rev. A 39, 438 (1989).
${ }^{15}$ P. Meakin, Ann. Rev. Phys. Chem. 39, 237 (1988).

${ }^{16}$ T. Nagatani, Phys. Rev. A 40, 7286 (1989).

${ }^{17}$ T. Vicsek, Phys. Rev. A 32, 3084 (1985).

${ }^{18}$ L. P. Kadanoff, J. Stat. Phys. 39, 267 (1985).

${ }^{19}$ R. Tao, M. A. Novotny, and K. Kashi, Phys. Rev. A 38, 1019 (1988).

20J. D. Sherwood, J. Phys. A 19, L195 (1986).

${ }^{21}$ P. R. King, J. Phys. A 20, L529 (1987).

${ }^{22}$ M. J. King and H. Scher, Phys. Rev. A 41, 874 (1990).

${ }^{23}$ J. Lee, A. Coniglio, and H. E. Stanley, Phys. Rev. A 41, 4589 (1990).

${ }^{24}$ R. F. Voss, J. Stat. Phys. 36, 861 (1984).

${ }^{25}$ M. Uwaha and Y. Saito, J. Phys. Soc. Jpn. 57, 3285 (1988).

${ }^{26}$ S. Miyajima, Y. Hasegawa, A. Bunde, and H. E. Stanley, J. Phys. Soc. Jpn. 57, 3376 (1988).

${ }^{27}$ P. Meakin, Phys. Rev. A 38, 994 (1988).

${ }^{28}$ R. F. Xiao, J. I. D. Alexander, and F. Rosenberger, Phys. Rev. A 39, 6397 (1989).

${ }^{29}$ D. Grier, E. Ben-Jacob, Roy Clarke, and L. M. Sander, Phys. Rev. Lett. 56, 1264 (1986).

${ }^{30}$ Y. Sawada, A. Dougherty, and J. P. Gollub, Phys. Rev. Lett. 56, 1260 (1986).

${ }^{31}$ P. Ramanlal and L. M. Sander, Phys. Rev. Lett. 54, 1828 (1985).

32J. Krug and P. Meakin, Phys. Rev. A 40, 2064 (1989).

${ }^{33}$ T. Nagatani, Phys. Rev. A 40, 5351 (1989). 